\title{
CASINO Monte Carlo Simulations of Scanning Transmission Electron Microscopy
}

\author{
H. Demers, ${ }^{*}$ N. Poirier-Demers, ${ }^{*}$ N. de Jonge, ${ }^{* *}, * * *$ and D. Drouin*
}

* Electrical and computer Engineering department, University of Sherbrooke, Sherbrooke, Quebec, J1k 2R1, Canada

** Department of Molecular Physiology and Biophysics, Vanderbilt University Medical Center, Nashville, 37232-0615, USA

*** Materials Science and Technology Division, Oak Ridge National Laboratory, Oak Ridge, TN 37831-6064, USA

The CASINO Monte Carlo software has been modified to include the simulation of a scanning transmission electron microscope (STEM) image acquired with the annular dark-field (ADF) detector. The electron trajectory core calculation models are based on the software CASINO v2 [1]. The software now includes electron-optical parameters, such as the semi-angle of the focused electron beam at the specimen, the opening angle of the ADF detector, the probe current, and the noise characteristics of the electron source. The modified CASINO code also includes the physical models of electron scattering in the energy range up to $300 \mathrm{kV}$. With the addition of this new electron-optical feature, it is now possible to scan the electron beam to acquire images or linescans at different focal points within the sample according to the depth of field of the simulated STEM. It is thus possible to simulate two- and three-dimensional (3D) datasets of samples of various geometries and chemical composition.

A novel STEM technique to characterize cells in a liquid compartment has been developed recently [2], so-called liquid STEM. A liquid specimen, e.g. water, is enclosed in a micro-fluidics chamber with electron-transparent silicon nitride membrane windows. CASINO was used to simulate STEM images of gold nanoparticles on water layers of several micrometers thickness. Fig. 1 shows a qualitative comparison of an experimental and a simulated STEM image of gold nanoparticles marker placed above a water layer of a thickness of $5 \mu \mathrm{m}$. A similar spatial resolution and contrast between the simulated and experiments images is observed. These experimental comparisons have been used to improve the simulations models by adding, for example, a more realistic electron source or electron optics. CASINO can thus be used to study the characteristics of STEM imaging of thick (for electron microscopy) specimens.

Another second series of simulations involved the recording of 3D datasets via focal-series. Taking advantage of the reduced depth of field of aberration-corrected STEM compared to standard STEM, 3D datasets can be recorded of biological specimens with an axial resolution in the range of several tens of nanometers [3]. Fig. 2a shows three simulated images of such 3D datasets at a focal position of $0 \mathrm{~nm}$ (top image), $500 \mathrm{~nm}$ (middle image) and $1000 \mathrm{~nm}$ (bottom image). The simulated sample consisted of three gold nanoparticles of $10 \mathrm{~nm}$ in diameter embedded in a carbon layer of $1 \mu \mathrm{m}$ in thickness. These nanoparticles were, from left to right, at the top $(0 \mathrm{~nm})$, middle $(500 \mathrm{~nm})$ and bottom $(1000 \mathrm{~nm})$ of the carbon layer. These images have been used to investigate the lateral resolution as a function of particle position within the layer and microscope settings (pixel dwell time, pixel size, focus step size, beam semi-angle, and beam energy). It is also possible to determine the vertical or depth resolution by simulating the detected intensity as a function of the focal position as shown in Fig. $2 b$. 


\section{References}

[1] D. Drouin et al., Scanning, 29 (2007) 92.

[2] N. de Jonge et al., Proc. Natl. Acad. Sci. 106 (2009) 2159.

[3] N. de Jonge et al., Microsc. Microanal. 16 (2010) 54.

[4] A Portion of this research was conducted at the SHaRE User Facility, which is sponsored by the Division of Scientific User Facilities, Office of Basic Energy Sciences, U.S. Department of Energy. This work was supported by NIH grant R01GM081801.
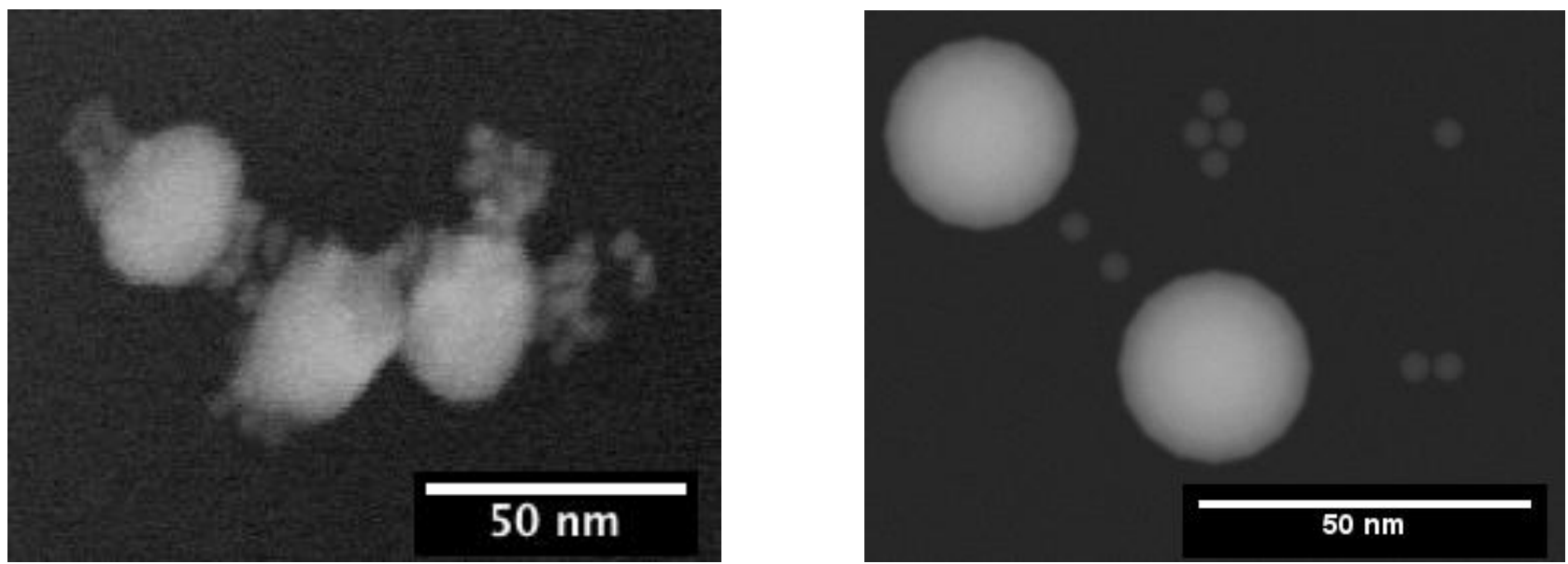

FIG. 1. Comparison of experimental (left) and simulated (right) STEM images of gold nanoparticles with diameters of $5 \mathrm{~nm}$ and $30 \mathrm{~nm}$ on top of a column of water of $5 \mu \mathrm{m}$ thickness enclosed between two silicon nitride windows of $50 \mathrm{~nm}$ in thickness each. The beam semi-angle was $11 \mathrm{mrad}$, the number of electrons per pixel was $72400(0.580 \mathrm{nA} \times 20 \mu \mathrm{s} / \mathrm{e})$, the beam voltage was $200 \mathrm{kV}$, and the ADF opening semi-angle was 94 mrad.

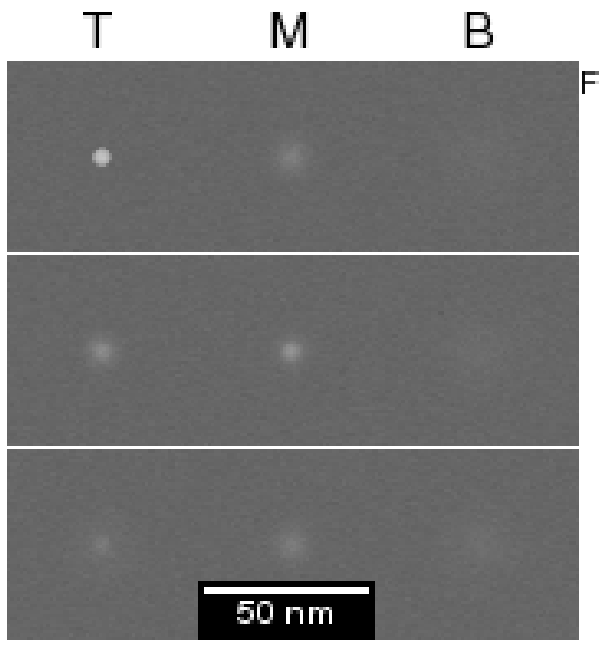

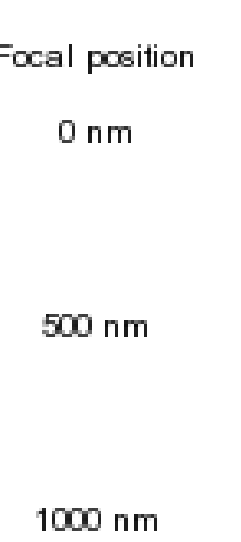

a)

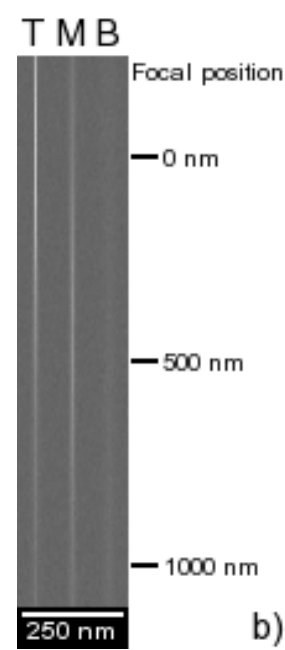

FIG. 2. 3D STEM images of $10 \mathrm{~nm}$ diameter gold nanoparticles embedded in a carbon film of $1 \mu \mathrm{m}$ thickness and placed at different depths (a) and (b) vertical linescan across the gold particles as a function of focal position. The beam semi-angle was $20 \mathrm{mrad}$, the number of electrons per pixel was 100 000, the beam voltage was $200 \mathrm{kV}$ and the ADF opening semi-angles was $50 \mathrm{mrad}$. 